“(C) 2015 IEEE. Personal use of this material is permitted. Permission from IEEE must be obtained for all other uses, in any current or future media, including reprinting/republishing this material for advertising or promotional purposes, creating new collective works, for resale or redistribution to servers or lists, or reuse of any copyrighted component of this work in other works." 


\title{
Augmented Reality based Illusion System with Biofeedback
}

\author{
Yee Mon Aung, Student Member, IEEE and Adel Al-Jumaily, Senior Member, IEEE
}

\begin{abstract}
This paper presents the Augmented Reality based Illusion System (ARIS) with biofeedback for upper limb rehabilitation. It aims for fast recovery of motor deficit with motivational approach over traditional upper limb rehabilitation methods. The system incorporates with Augmented Reality (AR) technology to develop upper limb rehabilitation exercise and computer vision with color recognition technique to create "Fool-the-Brain" concept for fast recovery of neural impairment due to various motor injuries. The rehabilitation exercise in ARIS is aiming to increase the shoulder joint range of motions by performing reaching movements and to strengthen the associated muscles. "Fool-the-Brain" concept is introduced during performing rehabilitation exercise to perceive artificial visual feedback where user's real impaired arm is covered by Virtual Arm (VA). When the real arm cannot perform the required task, VA will take over the job of real one and will make the user perceives the sense that is still be able to accomplish the task with own effort. Evaluation has performed and results indicate that ARIS with biofeedback is a potential upper limb rehabilitation system for people with upper limb motor deficit.
\end{abstract}

\section{INTRODUCTION}

Traumatic Brain Injury (TBI), Spinal Cord Injury (SCI) or Cerebrovascular Accident (CVA) leads to motor deficit which will result in loss of control over whole body or one side of the body depending on which part of the brain is affected. These injuries will limit a person to perform daily live activities and greatly impact his/her quality of life. Therefore, physical rehabilitations are required to perform for restoration of lost functions to promote the quality of life. According to the Physical Disability Australia (PDA), it was estimated that almost $15 \%$ of the Australia population have suffered from physical disabilities and will be more in future [1]. This demand leads to a shortage of physical therapists as traditional rehabilitation therapy requires one-to-one attention between patient and therapist. In addition to this, performing of traditional rehabilitation exercise will become mundane after long term training. Therefore, researchers have been trying to develop effective rehabilitation system which can require less supervision and fast recovery with motivational approach by employing various technologies that includes Augmented Reality (AR) for therapeutic purposes. AR technology has proven its effectiveness in the area of upper limb rehabilitation [2]. AR is the combination of real and virtual world where virtual objects lay on top of

\footnotetext{
Y.M.Aung is with the University of Technology Sydney, Australia, NSW 2007 (phone: +61 95142343; e-mail: yee.m.aung@student.uts.edu.au).

A. Al-Jumaily with University of Technology Sydney, Australia, NSW 2007, (e-mail: adel.al-jumaily@uts.edu.au)
}

the real world which is fed by webcam as a live video background image on the computer display screen. Therefore, this approach will provide better interactive training environment and more realistic. As far as AR based upper limb rehabilitation systems are concern, researchers from $[3,4]$ have developed AR based system to promote the reaching movements of upper limb and strengthen the associated muscles. Moving forward from this, researchers have tried to integrated with biofeedback system and it has proven that integrating with biofeedback system provides improvement in retraining of muscles strengthening [5]. The successful upper limb rehabilitation systems that integrated with biofeedback can be found in $[6,7]$.

In additional to that, researchers have found that the nature of human brain plasticity enhances fast recovery in rehabilitation due to its ability to alter in neural pathways by external stimulus. Taking the advantage of this fact, researchers have developed the mirror box therapy to treat the phantom limbs by creating the illusion scene to user perception [8]. The numbers of studies have proven that employing mirror box therapy which creates illusion scene enhance the motor deficit and reported has a positive results [9-11]. Recently this concept is becoming attractive for researchers to provide effective and fast recovery for motor rehabilitation. A group of researchers from [12] has studied and proved the effectiveness of artificial visual feedback by employing this concept. They defined this concept as "Foolthe-Brain" concept because their studies have proven that motor cortex activity was able to restore successfully via artificial visual feedback which is an illusory movement of paralyzed limb and able to fool the brain to improve phantom limb movement.

However, up to our knowledge, existing developments based on this concept are only developed for forearm and/or hand rehabilitation with mirror box to attain the artificial visual feedback. Therefore, we have developed pioneering system for rehabilitates the whole arm that integrates with artificial visual feedback by means of "Fool-the-Brain" concept based on AR technology and integrate with biofeedback system for fast recovery.

The remainder of this paper is organized as follows. Section 2 provides with system implementation which comprises with development of augmented reality based illusion system (ARIS) and integrating with biofeedback system. Section 3 presents the usability study and discuss the preliminary results. Conclusions and future works are discussed in Section 4. 


\section{SYSTEM IMPLEMENTATION}

ARIS has developed in Adobe Flash platform through ActionScrip3 framework. Papervision 3D library is used in order to track the multiple colors via any built-in camera or USB web camera and import 3D model of Virtual Arm (VA) to flash platform. The complete system is made up of Personal Computer (PC), USB webcam, two colour markers and FlexComp biofeedback system from Thought Technology [13].

\section{A. Augmented Reality based Illusion System (ARIS)}

Augmented Reality based Illusion System (ARIS) adapts "Fool-the-Brain" concept to perceive artificial visual feedback to improve impaired arm movements by taking advantage of human brain plasticity nature. ARIS is made up of four modules: multicolor tracking module, Virtual Arm (VA) module, Kinematic module, Fool-the-Brain module and Rehabilitation Exercise module as shown in Fig. 1.

- Multicolor tracking module: This module engages with Papervision 3D library to detect and track the multiple color markers which are attached to the user's shoulder and hand in real-time via webcam as shown in Fig. 1(a). It also handles for importing of 3D VA model from blender platform to flash platform as ".dae" format. Afterward, the imported model attaches to the shoulder color marker and overlaps user's real arm to perceive artificial visual appearance. The color marker at hand will interact with virtual objects to perform rehabilitation exercise that developed in rehabilitation exercise module. At the same time, the movement of the hand will be tracked in real-time and the performance of the hand movement displays on real-time trajectory graph as an immediate feedback to the therapist.

- Virtual Arm (VA) module: In this module, 3D VA model is built with blender software as portrayed in Fig. 1(b). The model arm segments are properly defined to ensure any motion of the parent link will have an effect on the child link during simulation. Defining of pivot point on each link is very important as this defines the centre of rotation of the link joint and it is carefully defined in developed VA. There are 6 DOFs in developed VA model where shoulder joint is limited to 3DOFs, elbow, forearm and wrist joint is modeled as cylindrical joint which allows the flexion/extension of the elbow and wrist and internal rotation of the forearm with
1DOF each.

- Kinematic module: In this kinematic module, mathematical modeling of VA is formulated with Denavit-Hartenberg (D-H) convention as illustrated in Fig. 1(c). The D-H parameters: a, $\alpha, \mathrm{d}$ and $\theta$ which represents the link length, link twist, link offset and joint angle, respectively are defined at respective joint in VA model. Moreover, VA joint rotations are limited to human arm safe articulation range for realistic movements. The values of such VA articulation range and $\mathrm{D}-\mathrm{H}$ parameters are presented in Table 1. According to the predefined trajectory which is developed in rehabilitation exercise, forward and inverse kinematics calculation is carried out to simulate VA model.

- "Fool-the-Brain" module: In this module, VA model overlays on top of the real impaired arm with the aid of multicolor tracking module. This overlaid model will allow the user to perceive as his/her own arm on the display screen. To create such illusion environment, four layers are created as shown in Fig. 1(d). The very first layer is fed with live video to create AR environment. In second layer, rectangular shape with rounded edges object (oval shape) is display. In this layer, webcam captured image is imported. However, this imported image can be only seen inside of that rectangular and the rest of the region is set as transparent. This is yield for removing of real arm on the display screen. The placement of the oval shape object is attached to color marker at shoulder. Therefore, current position of the real arm will be always tracked and overlapped with oval shape object wherever user's arm moves. In third layer, AR based upper limb rehabilitation exercise is overlaid. In fourth layer, VA will be overlaid on top of the oval shape object and create the illusion scene of real arm. When the user cannot accomplish the required task due to his/her limited motor movement, VA will take over the job of real arm as if user is still performing the reaching task. This appearance on the screen will fool the user scene that he/she is still able to perform the reaching exercise by his/her own effort to the destination point.

- Rehabilitation exercise module: This module is developed based on AR technology to enhance user motivation. Circular Motion Exercise (CME) is developed where its trajectory is chosen as a circular

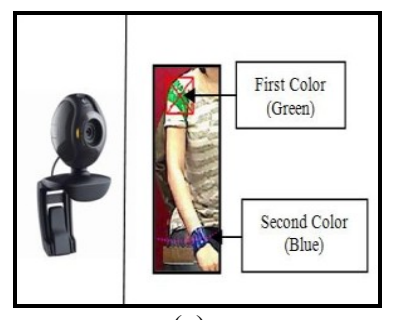

(a)

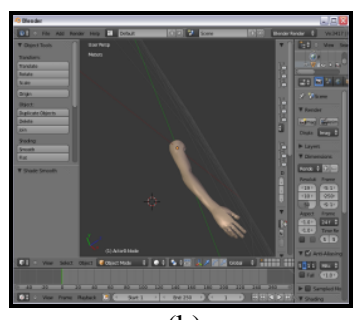

(b)

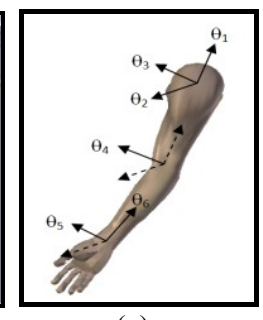

(c)

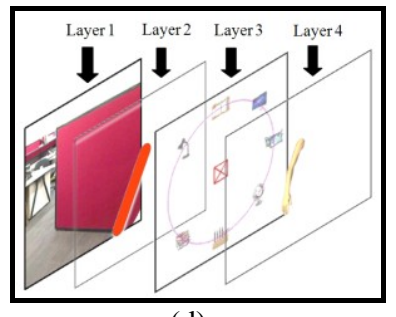

(d)

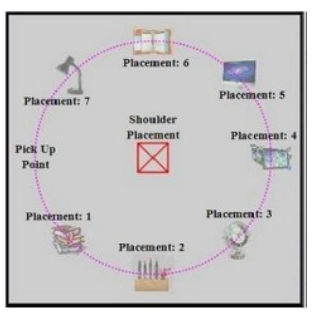

(e)

Figure 1. Individual module in ARIS (a) Multicolor tracking (b) Virtual Arm (VA) (c) Kinematic (d) "Fool-the-Brain" (e) Rehabilitation Exericse 
movement, as depicted in Fig. 1(e), because this movement will provide the widest range of motion at shoulder joint such as shoulder flexion, adduction and abduction, internal and external rotation. The aim of $\mathrm{CME}$ is to collect the virtual objects with color marker which attach to the user's hand and place them on the respective translucent virtual objects that are displayed on the screen. There are total of seven virtual objects that user requires to pick and place [14]. By performing of this exercise, user's anterior deltoid, posterior deltoid, biceps brachii and upper trapezius muscle will be trained and strengthened.

\section{B. Surface Electromyography (sEMG) Biofeedback}

Electromyography (sEMG) is one of the most important biological signals to monitor the performance of skeletal muscle. It can be acquired from the skin and known as surface EMG (sEMG). In this development, four sEMG signals were recorded and monitored from user's anterior deltoid, posterior deltoid, bicep brachii and upper trapezius muscles via FlexComp biofeedback system. The locations of the electrode sites are shown in Fig. 2. Extracted signals are then displayed in real-time on the screen and also recorded to analyze in future. Integrating with biofeedback in ARIS serves two main purposes for user and physiotherapist respectively. From the user point of view, displaying of realtime contributed muscle performance aims as one kind of motivational approach to visualize how the muscles are working during performing the exercise. From physical therapist point of view, it aims to monitor and track the muscle performances from real-time and recorded signals.

\section{USABILITY STUDY AND DISCUSSION}

Usability study was conducted with five able-bodied users and each person playing ARIS twice as shown in Fig. 3. After performing exercise in ARIS, users were requested to answer the questionnaire based on a visual-analogue likert scale according to the Heuristics evaluation [15] where " 1 "

TABLE I. D-H PARAMETERS AND ANATOMICAL MOTION AND LIMIT

\begin{tabular}{|c|c|c|c|c|c|c|c|}
\hline \multicolumn{2}{|c|}{ Joint } & Motion & $\boldsymbol{\alpha}$ & $\mathbf{a}$ & $\boldsymbol{\theta}$ & $\mathbf{d}$ & Joint Limit \\
\hline 1 & \multirow{3}{*}{ Shoulder } & Internal/External Rotation & $-\pi / 2$ & 0 & $\theta_{1}$ & 0 & $\left(-90^{\circ}, 30^{\circ}\right)$ \\
\cline { 3 - 8 } & & Abduction/Adduction & $\pi / 2$ & 0 & $\theta_{2}$ & 0 & $\left(-40^{\circ}, 180^{\circ}\right)$ \\
\cline { 3 - 8 } & & Flexion/Extension & 0 & $\mathrm{~L} 3$ & $\theta_{3}$ & 0 & $\left(0^{\circ}, 180^{\circ}\right)$ \\
\hline 3 & Elbow & Flexion/Extension & 0 & $\mathrm{~L} 4$ & $\theta_{4}$ & 0 & $\left(0^{\circ}, 130^{\circ}\right)$ \\
\hline 5 & Wrist & Flexion/Extension & $\pi / 2$ & 0 & $\theta_{5}$ & 0 & $\left(-45^{\circ}, 45^{\circ}\right)$ \\
\hline 6 & Forearm & Internal/External Rotation & 0 & $\mathrm{~L} 6$ & $\theta_{6}$ & 0 & $\left(0^{\circ}, 160^{\circ}\right)$ \\
\hline
\end{tabular}

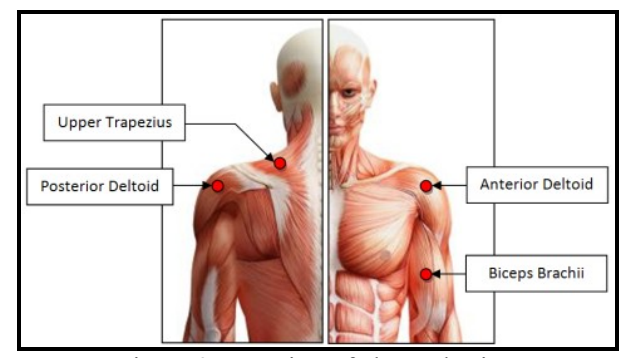

Figure 2. Location of electrode sites refers to strongly disagree and " 5 " refers to strongly agree. From the questionnaire results, all participants enjoyed to perform the exercise with "Fool-the-Brain" concept. Although most of the participants had not tried augmented reality based exercise before, they found that ARIS is very easy to understand, adapt and manipulate the system. All of the participants were happy to observe their hands movement in real-time arm trajectory graph and muscle activation in real-time. Most of the participants were immersed in the augmented reality environment and perceive good artificial visual feedback. However, one of the participants pointed out that it would be better to have auto detection of the radius of circular trajectory based on user whole arm length. The majority of the participants were happy with the color markers except one that suggested using color glove instead of wrist band to attain more convenient movement of hand. One participant felt the muscle fatigue during performing CME exercise. The results of the questionnaire are as depicted in Fig 4.

In addition to that, integrating with biofeedback system allowed all participants to monitor the actual muscles contribution and their performances during performing the exercise in real-time were recorded in Fig. 5. From the results, most of the participants have similar muscle contribution except subject 5's upper trapezius signal which was very high as shown in Fig. 5(e). This is because the subject felt muscle fatigue during performing CME and admitted that he did not take a good rest before participating in usability study. From the recorded signals, it is clearly showed that CME exercise is able to adapt to it easily just after second time of practice. All participants were enjoyed with the integrating as they are able to observe which muscles involve in which type of movement and they felt very motivated to complete the exercise. From the recorded signals from all participants, the anterior deltoid is the most

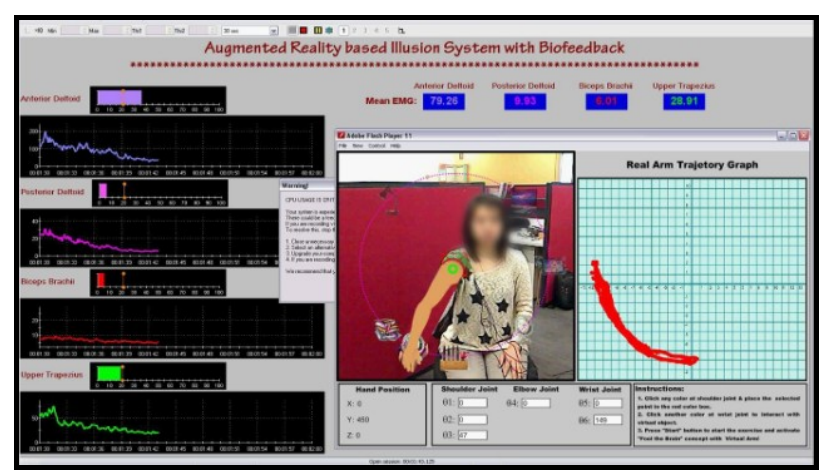

Figure 3. Usability Test with ARIS

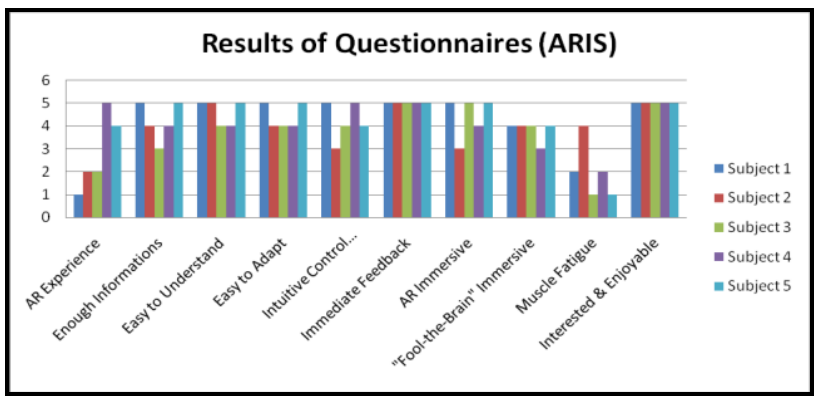

Figure 4. Questionnaire Results 


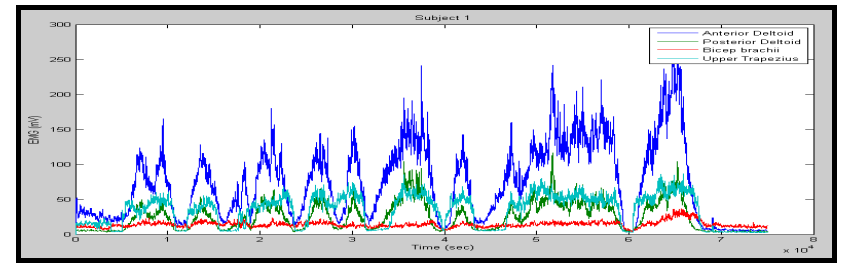

(a)

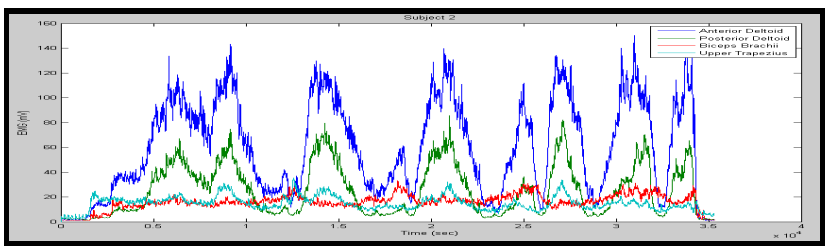

(b)

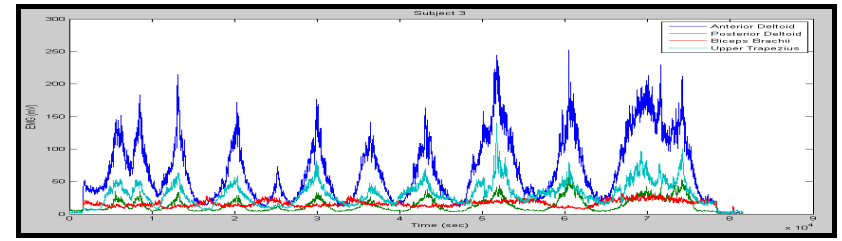

(c)

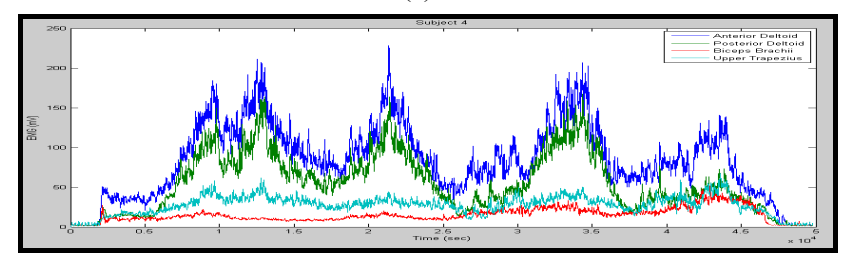

(d)

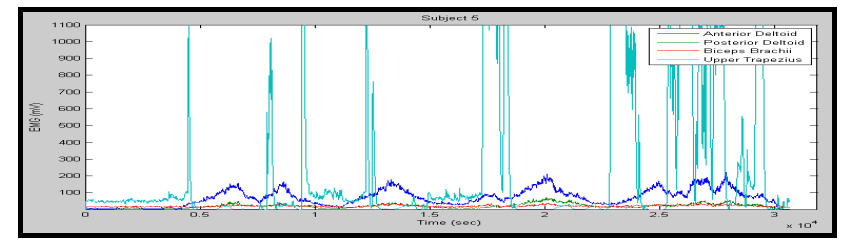

(e)

Figure 5. Muscle performance of (a) Subject 1 (b) Subject 2 (c) Subject 3 (d) Subject 4 and (e) Subject 5 during the usability test with ARIS

(Legend: Blue: Anterior Deltoid, Green: Posterior Deltoid, Red: Biceps Brachii, Cyan: Upper Trapezius)

contributed muscle to perform CME exercise followed by posterior deltoid and then upper trapeizus muscle. However the contribution of biceps brachii is very less in ARIS exercise. This information indicates that performing of CME exercise in ARIS strengthen the anterior deltoid, posterior deltoid and upper trapezius muscles. Therefore, it can be clearly seen that ARIS with biofeedback system achieves positive feedback and it has a potential to be used as a rehabilitation system although there are some rooms to improve according to the feedback from questionnaire.

\section{CONCLUSION AND FUTURE WORK}

In this paper, ARIS with biofeedback system which integrates with "Fool-the-Brain" concept, AR based rehabilitation exercise and biofeedback that aims for fast recovery of upper limb motor deficits with motivational approach had presented. The system has analyzed via
Heuristics evaluation and it showed encouraging results with positive feedback. This feedback indicates that the develop system is appropriate as a rehabilitation system for people with upper limb motor deficit. However, there are few improvements that require to be done according to the feedback from questionnaires and the work is underway to make these improvements. In addition, control method of VA model will be replaced with human intended movement by making use of recorded user's sEMG signals to become more effective rehabilitation system. After improvements have completed, clinical trial will be conducted in Port Kembla Rehabilitation Hospital in Australia and results will be reported in near future.

\section{REFERENCES}

[1] Physical Disability Australia. (2013, October). What is physical disability? Available: http://www.pda.org.au/what-is-physicaldisability

[2] H. Al-Issa, H. Regenbrecht, and L. Hale, "Augmented reality applications in rehabilitation to improve physical outcomes," Physical Therapy Reviews, vol. 17, pp. 16-28, 2012.

[3] Y. M. Aung and A. Al-Jumaily, "AR based upper limb rehabilitation system," in 4th IEEE RAS \& EMBS International Conference on Biomedical Robotics and Biomechatronics (BioRob), Rome, Italy, 2012, pp. 213-218.

[4] A. Dinevan, Y. M. Aung, and A. Al-Jumaily, "Human computer interactive system for fast recovery based stroke rehabilitation," in 11th International Conference on Hybrid Intelligent Systems (HIS), 2011, pp. 647-652.

[5] H. Huang, S. Wolf, and J. He, "Recent developments in biofeedback for neuromotor rehabilitation," Journal of NeuroEngineering and Rehabilitation, vol. 3, p. 11, 2006.

[6] Y. M. Aung and A. Al-Jumaily, "Shoulder rehabilitation with biofeedback simulation," in 2012 International Conference on Mechatronics and Automation (ICMA), Chengdu, China, 2012, pp. 974-979.

[7] Y. M. Aung and A. Al-Jumaily, "Neuromotor Rehabilitation System with Real-Time Biofeedback," International Journal of Computer Information Systems and Industrial Management Applications, vol. 5, pp. 550-556, 2013.

[8] K. C. Lin, Y. T. Chen, P. C. Huang, C. Y. Wu, W. L. Huang, H. W. Yang, H. T. Lai, and H. J. Lu, "Effect of mirror therapy combined with somatosensory stimulation on motor recovery and daily function in stroke patients: A pilot study," Journal of the Formosan Medical Association, 2012.

[9] H. Thieme, J. Mehrholz, M. Pohl, J. Behrens, and C. Dohle, "Mirror therapy for improving motor function after stroke," Stroke, vol. 44, pp. e1-e2, 2013.

[10] M. M. Lee, H.-y. Cho, and C. H. Song, "The Mirror Therapy Program Enhances Upper-Limb Motor Recovery and Motor Function in Acute Stroke Patients," American Journal of Physical Medicine \& Rehabilitation, vol. 91, pp. 689-700, 2012.

[11] H. Regenbrecht, S. Hoermann, G. McGregor, B. Dixon, E. Franz, C. Ott, L. Hale, T. Schubert, and J. Hoermann, "Visual manipulations for motor rehabilitation," Computers \& Graphics, vol. 36, pp. 819-834, 2012.

[12] P. Giraux and A. Sirigu, "Illusory movements of the paralyzed limb restore motor cortex activity," NeuroImage, vol. 20, Supplement 1, pp. S107-S111, 2003.

[13] Thought Techonology. (2013, October). FlexComp Infiniti. Available: http://www.thoughttechnology.com/

[14] Y. M. Aung and A. Al-Jumaily, "Illusion Approach for Upper Limb Motor Rehabilitation," in International Congress on Neurotechnology, Electronics and Informatics (NEUROTECHNIX 2013), Vilamoura, Algarve, Portugal, 2013, pp. 99-105. 
[15] H. Desurvire, M. Caplan, and J. A. Toth, "Using heuristics to evaluate the playability of games," in CHI '04 Extended Abstracts on Human Factors in Computing Systems, Vienna, Austria, 2004, pp. 15091512. 


\title{
Augmented Reality based Illusion System with Biofeedback
}

\author{
Yee Mon Aung, Student Member, IEEE and Adel Al-Jumaily, Senior Member, IEEE
}

\begin{abstract}
This paper presents the Augmented Reality based Illusion System (ARIS) with biofeedback for upper limb rehabilitation. It aims for fast recovery of motor deficit with motivational approach over traditional upper limb rehabilitation methods. The system incorporates with Augmented Reality (AR) technology to develop upper limb rehabilitation exercise and computer vision with color recognition technique to create "Fool-the-Brain" concept for fast recovery of neural impairment due to various motor injuries. The rehabilitation exercise in ARIS is aiming to increase the shoulder joint range of motions by performing reaching movements and to strengthen the associated muscles. "Fool-the-Brain" concept is introduced during performing rehabilitation exercise to perceive artificial visual feedback where user's real impaired arm is covered by Virtual Arm (VA). When the real arm cannot perform the required task, VA will take over the job of real one and will make the user perceives the sense that is still be able to accomplish the task with own effort. Evaluation has performed and results indicate that ARIS with biofeedback is a potential upper limb rehabilitation system for people with upper limb motor deficit.
\end{abstract}

\section{INTRODUCTION}

Traumatic Brain Injury (TBI), Spinal Cord Injury (SCI) or Cerebrovascular Accident (CVA) leads to motor deficit which will result in loss of control over whole body or one side of the body depending on which part of the brain is affected. These injuries will limit a person to perform daily live activities and greatly impact his/her quality of life. Therefore, physical rehabilitations are required to perform for restoration of lost functions to promote the quality of life. According to the Physical Disability Australia (PDA), it was estimated that almost $15 \%$ of the Australia population have suffered from physical disabilities and will be more in future [1]. This demand leads to a shortage of physical therapists as traditional rehabilitation therapy requires one-to-one attention between patient and therapist. In addition to this, performing of traditional rehabilitation exercise will become mundane after long term training. Therefore, researchers have been trying to develop effective rehabilitation system which can require less supervision and fast recovery with motivational approach by employing various technologies that includes Augmented Reality (AR) for therapeutic purposes. AR technology has proven its effectiveness in the area of upper limb rehabilitation [2]. AR is the combination of real and virtual world where virtual objects lay on top of

\footnotetext{
Y.M.Aung is with the University of Technology Sydney, Australia, NSW 2007 (phone: +61 95142343; e-mail: yee.m.aung@student.uts.edu.au).

A. Al-Jumaily with University of Technology Sydney, Australia, NSW 2007, (e-mail: adel.al-jumaily@uts.edu.au)
}

the real world which is fed by webcam as a live video background image on the computer display screen. Therefore, this approach will provide better interactive training environment and more realistic. As far as AR based upper limb rehabilitation systems are concern, researchers from $[3,4]$ have developed AR based system to promote the reaching movements of upper limb and strengthen the associated muscles. Moving forward from this, researchers have tried to integrated with biofeedback system and it has proven that integrating with biofeedback system provides improvement in retraining of muscles strengthening [5]. The successful upper limb rehabilitation systems that integrated with biofeedback can be found in $[6,7]$.

In additional to that, researchers have found that the nature of human brain plasticity enhances fast recovery in rehabilitation due to its ability to alter in neural pathways by external stimulus. Taking the advantage of this fact, researchers have developed the mirror box therapy to treat the phantom limbs by creating the illusion scene to user perception [8]. The numbers of studies have proven that employing mirror box therapy which creates illusion scene enhance the motor deficit and reported has a positive results [9-11]. Recently this concept is becoming attractive for researchers to provide effective and fast recovery for motor rehabilitation. A group of researchers from [12] has studied and proved the effectiveness of artificial visual feedback by employing this concept. They defined this concept as "Foolthe-Brain" concept because their studies have proven that motor cortex activity was able to restore successfully via artificial visual feedback which is an illusory movement of paralyzed limb and able to fool the brain to improve phantom limb movement.

However, up to our knowledge, existing developments based on this concept are only developed for forearm and/or hand rehabilitation with mirror box to attain the artificial visual feedback. Therefore, we have developed pioneering system for rehabilitates the whole arm that integrates with artificial visual feedback by means of "Fool-the-Brain" concept based on AR technology and integrate with biofeedback system for fast recovery.

The remainder of this paper is organized as follows. Section 2 provides with system implementation which comprises with development of augmented reality based illusion system (ARIS) and integrating with biofeedback system. Section 3 presents the usability study and discuss the preliminary results. Conclusions and future works are discussed in Section 4. 


\section{SYSTEM IMPLEMENTATION}

ARIS has developed in Adobe Flash platform through ActionScrip3 framework. Papervision 3D library is used in order to track the multiple colors via any built-in camera or USB web camera and import 3D model of Virtual Arm (VA) to flash platform. The complete system is made up of Personal Computer (PC), USB webcam, two colour markers and FlexComp biofeedback system from Thought Technology [13].

\section{A. Augmented Reality based Illusion System (ARIS)}

Augmented Reality based Illusion System (ARIS) adapts "Fool-the-Brain" concept to perceive artificial visual feedback to improve impaired arm movements by taking advantage of human brain plasticity nature. ARIS is made up of four modules: multicolor tracking module, Virtual Arm (VA) module, Kinematic module, Fool-the-Brain module and Rehabilitation Exercise module as shown in Fig. 1.

- Multicolor tracking module: This module engages with Papervision 3D library to detect and track the multiple color markers which are attached to the user's shoulder and hand in real-time via webcam as shown in Fig. 1(a). It also handles for importing of 3D VA model from blender platform to flash platform as ".dae" format. Afterward, the imported model attaches to the shoulder color marker and overlaps user's real arm to perceive artificial visual appearance. The color marker at hand will interact with virtual objects to perform rehabilitation exercise that developed in rehabilitation exercise module. At the same time, the movement of the hand will be tracked in real-time and the performance of the hand movement displays on real-time trajectory graph as an immediate feedback to the therapist.

- Virtual Arm (VA) module: In this module, 3D VA model is built with blender software as portrayed in Fig. 1(b). The model arm segments are properly defined to ensure any motion of the parent link will have an effect on the child link during simulation. Defining of pivot point on each link is very important as this defines the centre of rotation of the link joint and it is carefully defined in developed VA. There are 6 DOFs in developed VA model where shoulder joint is limited to 3DOFs, elbow, forearm and wrist joint is modeled as cylindrical joint which allows the flexion/extension of the elbow and wrist and internal rotation of the forearm with
1DOF each.

- Kinematic module: In this kinematic module, mathematical modeling of VA is formulated with Denavit-Hartenberg (D-H) convention as illustrated in Fig. 1(c). The D-H parameters: a, $\alpha, \mathrm{d}$ and $\theta$ which represents the link length, link twist, link offset and joint angle, respectively are defined at respective joint in VA model. Moreover, VA joint rotations are limited to human arm safe articulation range for realistic movements. The values of such VA articulation range and $\mathrm{D}-\mathrm{H}$ parameters are presented in Table 1. According to the predefined trajectory which is developed in rehabilitation exercise, forward and inverse kinematics calculation is carried out to simulate VA model.

- "Fool-the-Brain" module: In this module, VA model overlays on top of the real impaired arm with the aid of multicolor tracking module. This overlaid model will allow the user to perceive as his/her own arm on the display screen. To create such illusion environment, four layers are created as shown in Fig. 1(d). The very first layer is fed with live video to create AR environment. In second layer, rectangular shape with rounded edges object (oval shape) is display. In this layer, webcam captured image is imported. However, this imported image can be only seen inside of that rectangular and the rest of the region is set as transparent. This is yield for removing of real arm on the display screen. The placement of the oval shape object is attached to color marker at shoulder. Therefore, current position of the real arm will be always tracked and overlapped with oval shape object wherever user's arm moves. In third layer, AR based upper limb rehabilitation exercise is overlaid. In fourth layer, VA will be overlaid on top of the oval shape object and create the illusion scene of real arm. When the user cannot accomplish the required task due to his/her limited motor movement, VA will take over the job of real arm as if user is still performing the reaching task. This appearance on the screen will fool the user scene that he/she is still able to perform the reaching exercise by his/her own effort to the destination point.

- Rehabilitation exercise module: This module is developed based on AR technology to enhance user motivation. Circular Motion Exercise (CME) is developed where its trajectory is chosen as a circular

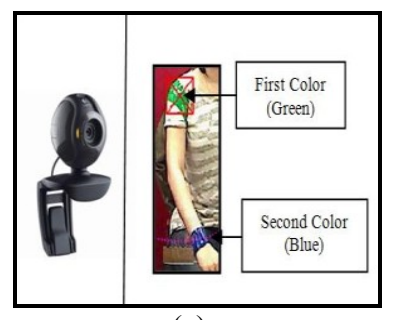

(a)

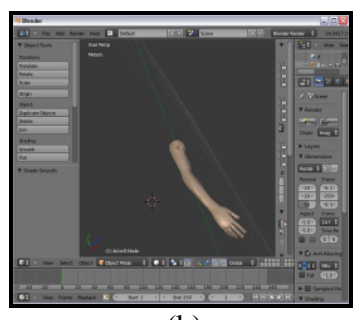

(b)

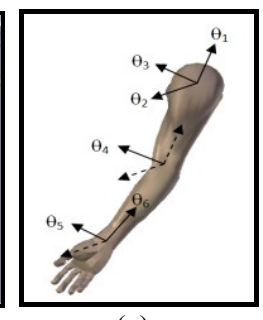

(c)

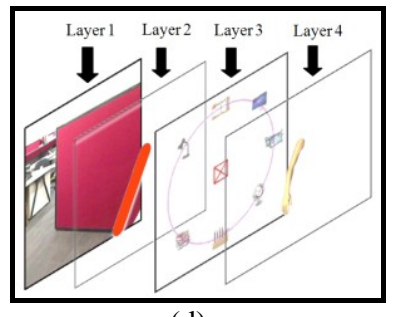

(d)

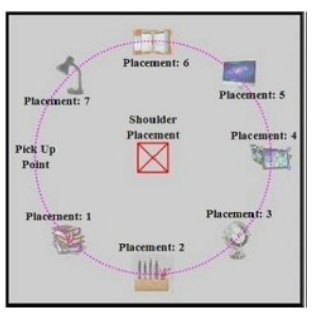

(e)

Figure 1. Individual module in ARIS (a) Multicolor tracking (b) Virtual Arm (VA) (c) Kinematic (d) "Fool-the-Brain" (e) Rehabilitation Exericse 
movement, as depicted in Fig. 1(e), because this movement will provide the widest range of motion at shoulder joint such as shoulder flexion, adduction and abduction, internal and external rotation. The aim of $\mathrm{CME}$ is to collect the virtual objects with color marker which attach to the user's hand and place them on the respective translucent virtual objects that are displayed on the screen. There are total of seven virtual objects that user requires to pick and place [14]. By performing of this exercise, user's anterior deltoid, posterior deltoid, biceps brachii and upper trapezius muscle will be trained and strengthened.

\section{B. Surface Electromyography (sEMG) Biofeedback}

Electromyography (sEMG) is one of the most important biological signals to monitor the performance of skeletal muscle. It can be acquired from the skin and known as surface EMG (sEMG). In this development, four sEMG signals were recorded and monitored from user's anterior deltoid, posterior deltoid, bicep brachii and upper trapezius muscles via FlexComp biofeedback system. The locations of the electrode sites are shown in Fig. 2. Extracted signals are then displayed in real-time on the screen and also recorded to analyze in future. Integrating with biofeedback in ARIS serves two main purposes for user and physiotherapist respectively. From the user point of view, displaying of realtime contributed muscle performance aims as one kind of motivational approach to visualize how the muscles are working during performing the exercise. From physical therapist point of view, it aims to monitor and track the muscle performances from real-time and recorded signals.

\section{USABILITY STUDY AND DISCUSSION}

Usability study was conducted with five able-bodied users and each person playing ARIS twice as shown in Fig. 3. After performing exercise in ARIS, users were requested to answer the questionnaire based on a visual-analogue likert scale according to the Heuristics evaluation [15] where " 1 "

TABLE I. D-H PARAMETERS AND ANATOMICAL MOTION AND LIMIT

\begin{tabular}{|c|c|c|c|c|c|c|c|}
\hline \multicolumn{2}{|c|}{ Joint } & Motion & $\boldsymbol{\alpha}$ & $\mathbf{a}$ & $\boldsymbol{\theta}$ & $\mathbf{d}$ & Joint Limit \\
\hline 1 & \multirow{3}{*}{ Shoulder } & Internal/External Rotation & $-\pi / 2$ & 0 & $\theta_{1}$ & 0 & $\left(-90^{\circ}, 30^{\circ}\right)$ \\
\cline { 3 - 8 } & & Abduction/Adduction & $\pi / 2$ & 0 & $\theta_{2}$ & 0 & $\left(-40^{\circ}, 180^{\circ}\right)$ \\
\cline { 3 - 8 } & & Flexion/Extension & 0 & $\mathrm{~L} 3$ & $\theta_{3}$ & 0 & $\left(0^{\circ}, 180^{\circ}\right)$ \\
\hline 3 & Elbow & Flexion/Extension & 0 & $\mathrm{~L} 4$ & $\theta_{4}$ & 0 & $\left(0^{\circ}, 130^{\circ}\right)$ \\
\hline 5 & Wrist & Flexion/Extension & $\pi / 2$ & 0 & $\theta_{5}$ & 0 & $\left(-45^{\circ}, 45^{\circ}\right)$ \\
\hline 6 & Forearm & Internal/External Rotation & 0 & $\mathrm{~L} 6$ & $\theta_{6}$ & 0 & $\left(0^{\circ}, 160^{\circ}\right)$ \\
\hline
\end{tabular}

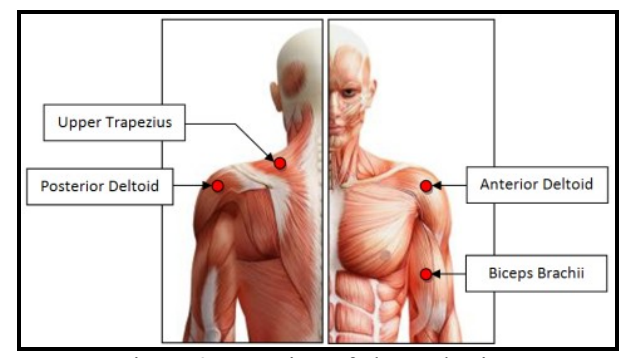

Figure 2. Location of electrode sites refers to strongly disagree and " 5 " refers to strongly agree. From the questionnaire results, all participants enjoyed to perform the exercise with "Fool-the-Brain" concept. Although most of the participants had not tried augmented reality based exercise before, they found that ARIS is very easy to understand, adapt and manipulate the system. All of the participants were happy to observe their hands movement in real-time arm trajectory graph and muscle activation in real-time. Most of the participants were immersed in the augmented reality environment and perceive good artificial visual feedback. However, one of the participants pointed out that it would be better to have auto detection of the radius of circular trajectory based on user whole arm length. The majority of the participants were happy with the color markers except one that suggested using color glove instead of wrist band to attain more convenient movement of hand. One participant felt the muscle fatigue during performing CME exercise. The results of the questionnaire are as depicted in Fig 4.

In addition to that, integrating with biofeedback system allowed all participants to monitor the actual muscles contribution and their performances during performing the exercise in real-time were recorded in Fig. 5. From the results, most of the participants have similar muscle contribution except subject 5's upper trapezius signal which was very high as shown in Fig. 5(e). This is because the subject felt muscle fatigue during performing CME and admitted that he did not take a good rest before participating in usability study. From the recorded signals, it is clearly showed that CME exercise is able to adapt to it easily just after second time of practice. All participants were enjoyed with the integrating as they are able to observe which muscles involve in which type of movement and they felt very motivated to complete the exercise. From the recorded signals from all participants, the anterior deltoid is the most

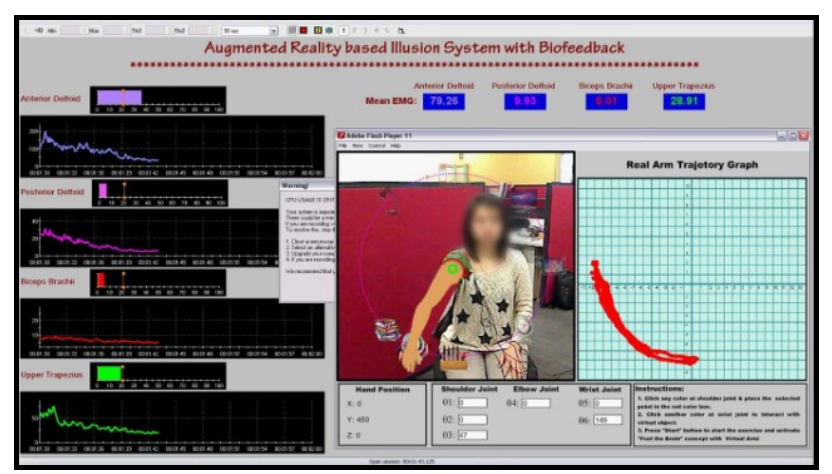

Figure 3. Usability Test with ARIS

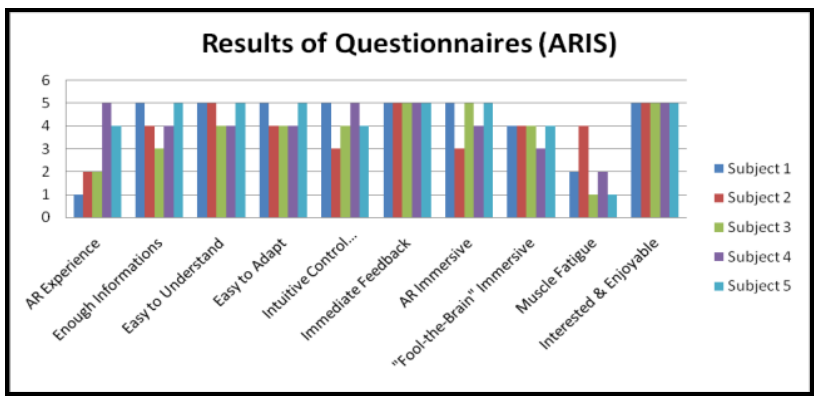

Figure 4. Questionnaire Results 


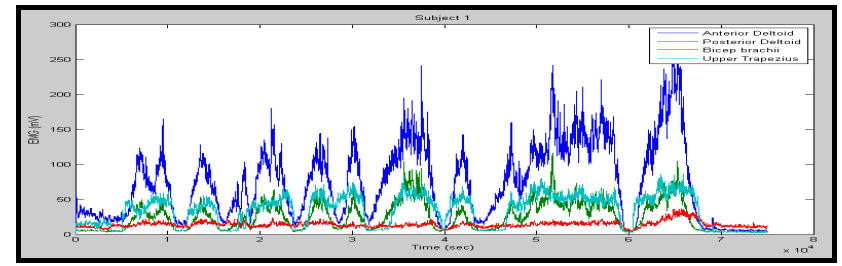

(a)

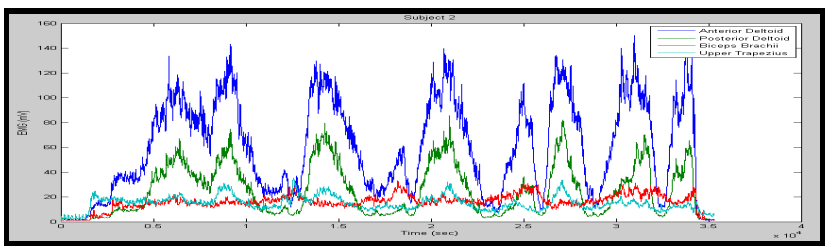

(b)

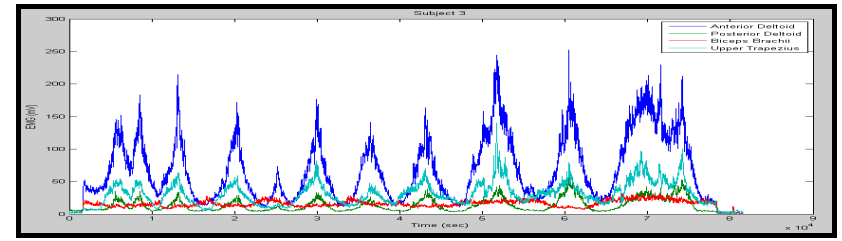

(c)

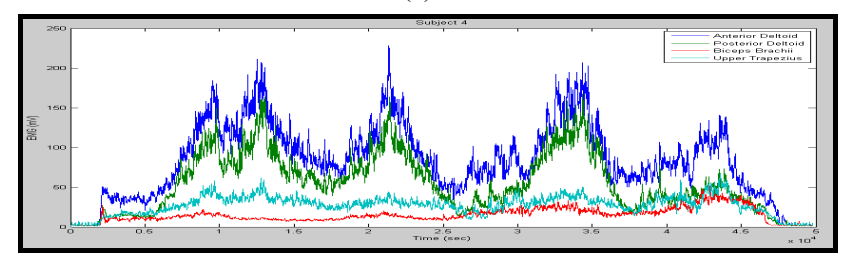

(d)

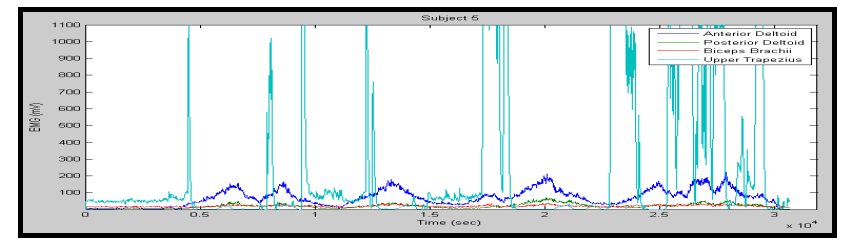

(e)

Figure 5. Muscle performance of (a) Subject 1 (b) Subject 2 (c) Subject 3 (d) Subject 4 and (e) Subject 5 during the usability test with ARIS

(Legend: Blue: Anterior Deltoid, Green: Posterior Deltoid, Red: Biceps Brachii, Cyan: Upper Trapezius)

contributed muscle to perform CME exercise followed by posterior deltoid and then upper trapeizus muscle. However the contribution of biceps brachii is very less in ARIS exercise. This information indicates that performing of CME exercise in ARIS strengthen the anterior deltoid, posterior deltoid and upper trapezius muscles. Therefore, it can be clearly seen that ARIS with biofeedback system achieves positive feedback and it has a potential to be used as a rehabilitation system although there are some rooms to improve according to the feedback from questionnaire.

\section{CONCLUSION AND FUTURE WORK}

In this paper, ARIS with biofeedback system which integrates with "Fool-the-Brain" concept, AR based rehabilitation exercise and biofeedback that aims for fast recovery of upper limb motor deficits with motivational approach had presented. The system has analyzed via
Heuristics evaluation and it showed encouraging results with positive feedback. This feedback indicates that the develop system is appropriate as a rehabilitation system for people with upper limb motor deficit. However, there are few improvements that require to be done according to the feedback from questionnaires and the work is underway to make these improvements. In addition, control method of VA model will be replaced with human intended movement by making use of recorded user's sEMG signals to become more effective rehabilitation system. After improvements have completed, clinical trial will be conducted in Port Kembla Rehabilitation Hospital in Australia and results will be reported in near future.

\section{REFERENCES}

[1] Physical Disability Australia. (2013, October). What is physical disability? Available: http://www.pda.org.au/what-is-physicaldisability

[2] H. Al-Issa, H. Regenbrecht, and L. Hale, "Augmented reality applications in rehabilitation to improve physical outcomes," Physical Therapy Reviews, vol. 17, pp. 16-28, 2012.

[3] Y. M. Aung and A. Al-Jumaily, "AR based upper limb rehabilitation system," in 4th IEEE RAS \& EMBS International Conference on Biomedical Robotics and Biomechatronics (BioRob), Rome, Italy, 2012, pp. 213-218.

[4] A. Dinevan, Y. M. Aung, and A. Al-Jumaily, "Human computer interactive system for fast recovery based stroke rehabilitation," in 11th International Conference on Hybrid Intelligent Systems (HIS), 2011, pp. 647-652.

[5] H. Huang, S. Wolf, and J. He, "Recent developments in biofeedback for neuromotor rehabilitation," Journal of NeuroEngineering and Rehabilitation, vol. 3, p. 11, 2006.

[6] Y. M. Aung and A. Al-Jumaily, "Shoulder rehabilitation with biofeedback simulation," in 2012 International Conference on Mechatronics and Automation (ICMA), Chengdu, China, 2012, pp. 974-979.

[7] Y. M. Aung and A. Al-Jumaily, "Neuromotor Rehabilitation System with Real-Time Biofeedback," International Journal of Computer Information Systems and Industrial Management Applications, vol. 5, pp. 550-556, 2013.

[8] K. C. Lin, Y. T. Chen, P. C. Huang, C. Y. Wu, W. L. Huang, H. W. Yang, H. T. Lai, and H. J. Lu, "Effect of mirror therapy combined with somatosensory stimulation on motor recovery and daily function in stroke patients: A pilot study," Journal of the Formosan Medical Association, 2012.

[9] H. Thieme, J. Mehrholz, M. Pohl, J. Behrens, and C. Dohle, "Mirror therapy for improving motor function after stroke," Stroke, vol. 44, pp. e1-e2, 2013.

[10] M. M. Lee, H.-y. Cho, and C. H. Song, "The Mirror Therapy Program Enhances Upper-Limb Motor Recovery and Motor Function in Acute Stroke Patients," American Journal of Physical Medicine \& Rehabilitation, vol. 91, pp. 689-700, 2012.

[11] H. Regenbrecht, S. Hoermann, G. McGregor, B. Dixon, E. Franz, C. Ott, L. Hale, T. Schubert, and J. Hoermann, "Visual manipulations for motor rehabilitation," Computers \& Graphics, vol. 36, pp. 819-834, 2012.

[12] P. Giraux and A. Sirigu, "Illusory movements of the paralyzed limb restore motor cortex activity," NeuroImage, vol. 20, Supplement 1, pp. S107-S111, 2003.

[13] Thought Techonology. (2013, October). FlexComp Infiniti. Available: http://www.thoughttechnology.com/

[14] Y. M. Aung and A. Al-Jumaily, "Illusion Approach for Upper Limb Motor Rehabilitation," in International Congress on Neurotechnology, Electronics and Informatics (NEUROTECHNIX 2013), Vilamoura, Algarve, Portugal, 2013, pp. 99-105. 
[15] H. Desurvire, M. Caplan, and J. A. Toth, "Using heuristics to evaluate the playability of games," in CHI '04 Extended Abstracts on Human Factors in Computing Systems, Vienna, Austria, 2004, pp. 15091512. 


\title{
Augmented Reality based Illusion System with Biofeedback
}

\author{
Yee Mon Aung, Student Member, IEEE and Adel Al-Jumaily, Senior Member, IEEE
}

\begin{abstract}
This paper presents the Augmented Reality based Illusion System (ARIS) with biofeedback for upper limb rehabilitation. It aims for fast recovery of motor deficit with motivational approach over traditional upper limb rehabilitation methods. The system incorporates with Augmented Reality (AR) technology to develop upper limb rehabilitation exercise and computer vision with color recognition technique to create "Fool-the-Brain" concept for fast recovery of neural impairment due to various motor injuries. The rehabilitation exercise in ARIS is aiming to increase the shoulder joint range of motions by performing reaching movements and to strengthen the associated muscles. "Fool-the-Brain" concept is introduced during performing rehabilitation exercise to perceive artificial visual feedback where user's real impaired arm is covered by Virtual Arm (VA). When the real arm cannot perform the required task, VA will take over the job of real one and will make the user perceives the sense that is still be able to accomplish the task with own effort. Evaluation has performed and results indicate that ARIS with biofeedback is a potential upper limb rehabilitation system for people with upper limb motor deficit.
\end{abstract}

\section{INTRODUCTION}

Traumatic Brain Injury (TBI), Spinal Cord Injury (SCI) or Cerebrovascular Accident (CVA) leads to motor deficit which will result in loss of control over whole body or one side of the body depending on which part of the brain is affected. These injuries will limit a person to perform daily live activities and greatly impact his/her quality of life. Therefore, physical rehabilitations are required to perform for restoration of lost functions to promote the quality of life. According to the Physical Disability Australia (PDA), it was estimated that almost $15 \%$ of the Australia population have suffered from physical disabilities and will be more in future [1]. This demand leads to a shortage of physical therapists as traditional rehabilitation therapy requires one-to-one attention between patient and therapist. In addition to this, performing of traditional rehabilitation exercise will become mundane after long term training. Therefore, researchers have been trying to develop effective rehabilitation system which can require less supervision and fast recovery with motivational approach by employing various technologies that includes Augmented Reality (AR) for therapeutic purposes. AR technology has proven its effectiveness in the area of upper limb rehabilitation [2]. AR is the combination of real and virtual world where virtual objects lay on top of

\footnotetext{
Y.M.Aung is with the University of Technology Sydney, Australia, NSW 2007 (phone: +61 95142343; e-mail: yee.m.aung@student.uts.edu.au).

A. Al-Jumaily with University of Technology Sydney, Australia, NSW 2007, (e-mail: adel.al-jumaily@uts.edu.au)
}

the real world which is fed by webcam as a live video background image on the computer display screen. Therefore, this approach will provide better interactive training environment and more realistic. As far as AR based upper limb rehabilitation systems are concern, researchers from $[3,4]$ have developed AR based system to promote the reaching movements of upper limb and strengthen the associated muscles. Moving forward from this, researchers have tried to integrated with biofeedback system and it has proven that integrating with biofeedback system provides improvement in retraining of muscles strengthening [5]. The successful upper limb rehabilitation systems that integrated with biofeedback can be found in $[6,7]$.

In additional to that, researchers have found that the nature of human brain plasticity enhances fast recovery in rehabilitation due to its ability to alter in neural pathways by external stimulus. Taking the advantage of this fact, researchers have developed the mirror box therapy to treat the phantom limbs by creating the illusion scene to user perception [8]. The numbers of studies have proven that employing mirror box therapy which creates illusion scene enhance the motor deficit and reported has a positive results [9-11]. Recently this concept is becoming attractive for researchers to provide effective and fast recovery for motor rehabilitation. A group of researchers from [12] has studied and proved the effectiveness of artificial visual feedback by employing this concept. They defined this concept as "Foolthe-Brain" concept because their studies have proven that motor cortex activity was able to restore successfully via artificial visual feedback which is an illusory movement of paralyzed limb and able to fool the brain to improve phantom limb movement.

However, up to our knowledge, existing developments based on this concept are only developed for forearm and/or hand rehabilitation with mirror box to attain the artificial visual feedback. Therefore, we have developed pioneering system for rehabilitates the whole arm that integrates with artificial visual feedback by means of "Fool-the-Brain" concept based on AR technology and integrate with biofeedback system for fast recovery.

The remainder of this paper is organized as follows. Section 2 provides with system implementation which comprises with development of augmented reality based illusion system (ARIS) and integrating with biofeedback system. Section 3 presents the usability study and discuss the preliminary results. Conclusions and future works are discussed in Section 4. 


\section{SYSTEM IMPLEMENTATION}

ARIS has developed in Adobe Flash platform through ActionScrip3 framework. Papervision 3D library is used in order to track the multiple colors via any built-in camera or USB web camera and import 3D model of Virtual Arm (VA) to flash platform. The complete system is made up of Personal Computer (PC), USB webcam, two colour markers and FlexComp biofeedback system from Thought Technology [13].

\section{A. Augmented Reality based Illusion System (ARIS)}

Augmented Reality based Illusion System (ARIS) adapts "Fool-the-Brain" concept to perceive artificial visual feedback to improve impaired arm movements by taking advantage of human brain plasticity nature. ARIS is made up of four modules: multicolor tracking module, Virtual Arm (VA) module, Kinematic module, Fool-the-Brain module and Rehabilitation Exercise module as shown in Fig. 1.

- Multicolor tracking module: This module engages with Papervision 3D library to detect and track the multiple color markers which are attached to the user's shoulder and hand in real-time via webcam as shown in Fig. 1(a). It also handles for importing of 3D VA model from blender platform to flash platform as ".dae" format. Afterward, the imported model attaches to the shoulder color marker and overlaps user's real arm to perceive artificial visual appearance. The color marker at hand will interact with virtual objects to perform rehabilitation exercise that developed in rehabilitation exercise module. At the same time, the movement of the hand will be tracked in real-time and the performance of the hand movement displays on real-time trajectory graph as an immediate feedback to the therapist.

- Virtual Arm (VA) module: In this module, 3D VA model is built with blender software as portrayed in Fig. 1(b). The model arm segments are properly defined to ensure any motion of the parent link will have an effect on the child link during simulation. Defining of pivot point on each link is very important as this defines the centre of rotation of the link joint and it is carefully defined in developed VA. There are 6 DOFs in developed VA model where shoulder joint is limited to 3DOFs, elbow, forearm and wrist joint is modeled as cylindrical joint which allows the flexion/extension of the elbow and wrist and internal rotation of the forearm with
1DOF each.

- Kinematic module: In this kinematic module, mathematical modeling of VA is formulated with Denavit-Hartenberg (D-H) convention as illustrated in Fig. 1(c). The D-H parameters: a, $\alpha, \mathrm{d}$ and $\theta$ which represents the link length, link twist, link offset and joint angle, respectively are defined at respective joint in VA model. Moreover, VA joint rotations are limited to human arm safe articulation range for realistic movements. The values of such VA articulation range and $\mathrm{D}-\mathrm{H}$ parameters are presented in Table 1. According to the predefined trajectory which is developed in rehabilitation exercise, forward and inverse kinematics calculation is carried out to simulate VA model.

- "Fool-the-Brain" module: In this module, VA model overlays on top of the real impaired arm with the aid of multicolor tracking module. This overlaid model will allow the user to perceive as his/her own arm on the display screen. To create such illusion environment, four layers are created as shown in Fig. 1(d). The very first layer is fed with live video to create AR environment. In second layer, rectangular shape with rounded edges object (oval shape) is display. In this layer, webcam captured image is imported. However, this imported image can be only seen inside of that rectangular and the rest of the region is set as transparent. This is yield for removing of real arm on the display screen. The placement of the oval shape object is attached to color marker at shoulder. Therefore, current position of the real arm will be always tracked and overlapped with oval shape object wherever user's arm moves. In third layer, AR based upper limb rehabilitation exercise is overlaid. In fourth layer, VA will be overlaid on top of the oval shape object and create the illusion scene of real arm. When the user cannot accomplish the required task due to his/her limited motor movement, VA will take over the job of real arm as if user is still performing the reaching task. This appearance on the screen will fool the user scene that he/she is still able to perform the reaching exercise by his/her own effort to the destination point.

- Rehabilitation exercise module: This module is developed based on AR technology to enhance user motivation. Circular Motion Exercise (CME) is developed where its trajectory is chosen as a circular

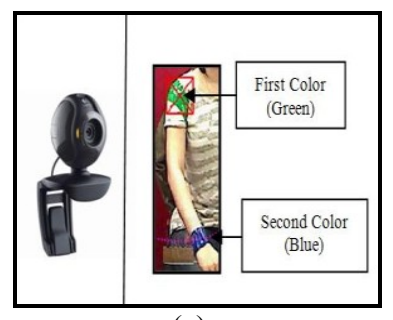

(a)

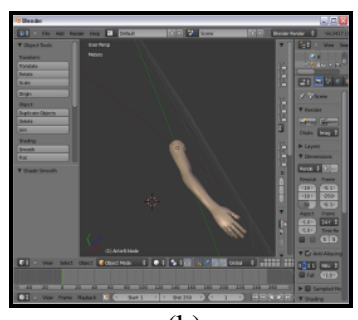

(b)

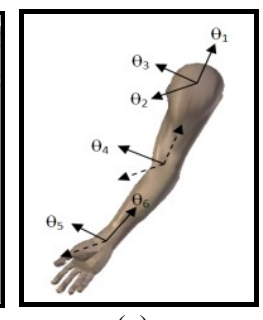

(c)

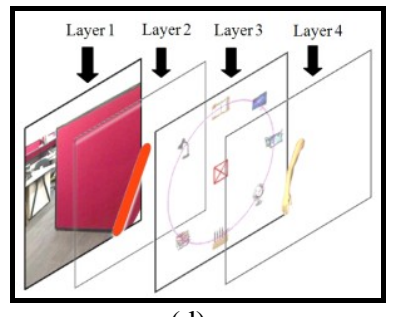

(d)

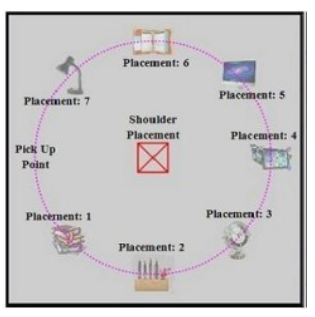

(e)

Figure 1. Individual module in ARIS (a) Multicolor tracking (b) Virtual Arm (VA) (c) Kinematic (d) "Fool-the-Brain" (e) Rehabilitation Exericse 
movement, as depicted in Fig. 1(e), because this movement will provide the widest range of motion at shoulder joint such as shoulder flexion, adduction and abduction, internal and external rotation. The aim of $\mathrm{CME}$ is to collect the virtual objects with color marker which attach to the user's hand and place them on the respective translucent virtual objects that are displayed on the screen. There are total of seven virtual objects that user requires to pick and place [14]. By performing of this exercise, user's anterior deltoid, posterior deltoid, biceps brachii and upper trapezius muscle will be trained and strengthened.

\section{B. Surface Electromyography (sEMG) Biofeedback}

Electromyography (sEMG) is one of the most important biological signals to monitor the performance of skeletal muscle. It can be acquired from the skin and known as surface EMG (sEMG). In this development, four sEMG signals were recorded and monitored from user's anterior deltoid, posterior deltoid, bicep brachii and upper trapezius muscles via FlexComp biofeedback system. The locations of the electrode sites are shown in Fig. 2. Extracted signals are then displayed in real-time on the screen and also recorded to analyze in future. Integrating with biofeedback in ARIS serves two main purposes for user and physiotherapist respectively. From the user point of view, displaying of realtime contributed muscle performance aims as one kind of motivational approach to visualize how the muscles are working during performing the exercise. From physical therapist point of view, it aims to monitor and track the muscle performances from real-time and recorded signals.

\section{USABILITY STUDY AND DISCUSSION}

Usability study was conducted with five able-bodied users and each person playing ARIS twice as shown in Fig. 3. After performing exercise in ARIS, users were requested to answer the questionnaire based on a visual-analogue likert scale according to the Heuristics evaluation [15] where " 1 "

TABLE I. D-H PARAMETERS AND ANATOMICAL MOTION AND LIMIT

\begin{tabular}{|c|c|c|c|c|c|c|c|}
\hline \multicolumn{2}{|c|}{ Joint } & Motion & $\boldsymbol{\alpha}$ & $\mathbf{a}$ & $\boldsymbol{\theta}$ & $\mathbf{d}$ & Joint Limit \\
\hline 1 & \multirow{3}{*}{ Shoulder } & Internal/External Rotation & $-\pi / 2$ & 0 & $\theta_{1}$ & 0 & $\left(-90^{\circ}, 30^{\circ}\right)$ \\
\cline { 3 - 8 } & & Abduction/Adduction & $\pi / 2$ & 0 & $\theta_{2}$ & 0 & $\left(-40^{\circ}, 180^{\circ}\right)$ \\
\cline { 3 - 8 } & & Flexion/Extension & 0 & $\mathrm{~L} 3$ & $\theta_{3}$ & 0 & $\left(0^{\circ}, 180^{\circ}\right)$ \\
\hline 3 & Elbow & Flexion/Extension & 0 & $\mathrm{~L} 4$ & $\theta_{4}$ & 0 & $\left(0^{\circ}, 130^{\circ}\right)$ \\
\hline 5 & Wrist & Flexion/Extension & $\pi / 2$ & 0 & $\theta_{5}$ & 0 & $\left(-45^{\circ}, 45^{\circ}\right)$ \\
\hline 6 & Forearm & Internal/External Rotation & 0 & $\mathrm{~L} 6$ & $\theta_{6}$ & 0 & $\left(0^{\circ}, 160^{\circ}\right)$ \\
\hline
\end{tabular}

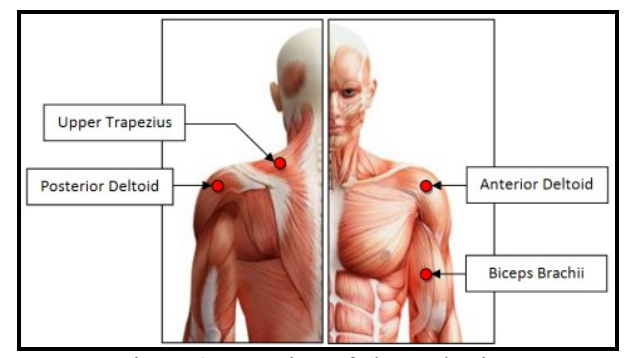

Figure 2. Location of electrode sites refers to strongly disagree and " 5 " refers to strongly agree. From the questionnaire results, all participants enjoyed to perform the exercise with "Fool-the-Brain" concept. Although most of the participants had not tried augmented reality based exercise before, they found that ARIS is very easy to understand, adapt and manipulate the system. All of the participants were happy to observe their hands movement in real-time arm trajectory graph and muscle activation in real-time. Most of the participants were immersed in the augmented reality environment and perceive good artificial visual feedback. However, one of the participants pointed out that it would be better to have auto detection of the radius of circular trajectory based on user whole arm length. The majority of the participants were happy with the color markers except one that suggested using color glove instead of wrist band to attain more convenient movement of hand. One participant felt the muscle fatigue during performing CME exercise. The results of the questionnaire are as depicted in Fig 4.

In addition to that, integrating with biofeedback system allowed all participants to monitor the actual muscles contribution and their performances during performing the exercise in real-time were recorded in Fig. 5. From the results, most of the participants have similar muscle contribution except subject 5's upper trapezius signal which was very high as shown in Fig. 5(e). This is because the subject felt muscle fatigue during performing CME and admitted that he did not take a good rest before participating in usability study. From the recorded signals, it is clearly showed that CME exercise is able to adapt to it easily just after second time of practice. All participants were enjoyed with the integrating as they are able to observe which muscles involve in which type of movement and they felt very motivated to complete the exercise. From the recorded signals from all participants, the anterior deltoid is the most

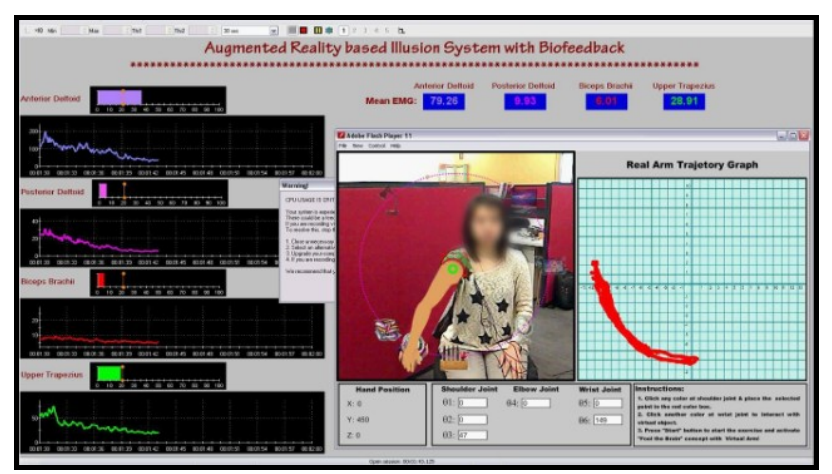

Figure 3. Usability Test with ARIS

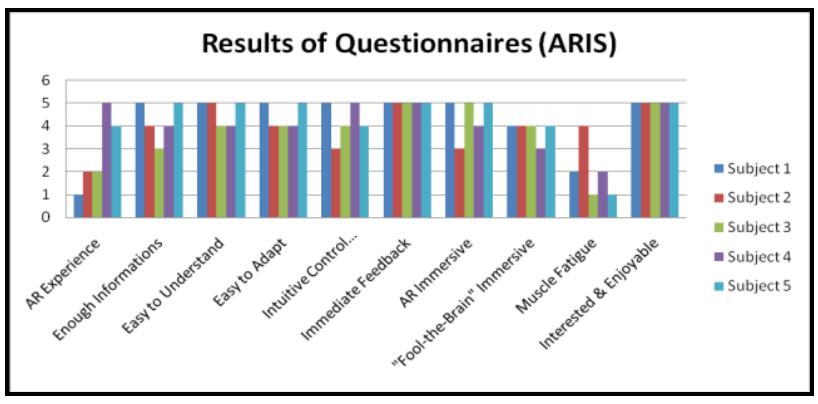

Figure 4. Questionnaire Results 


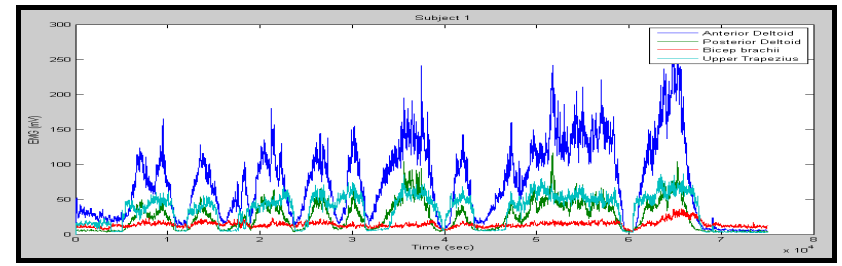

(a)

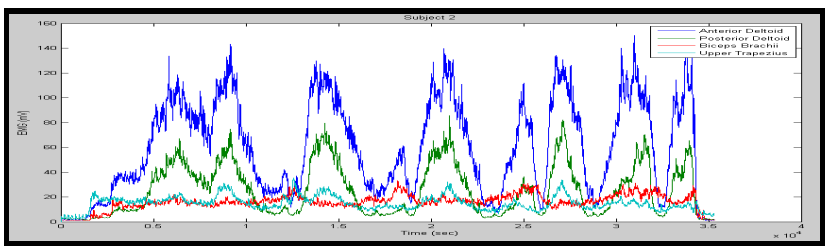

(b)

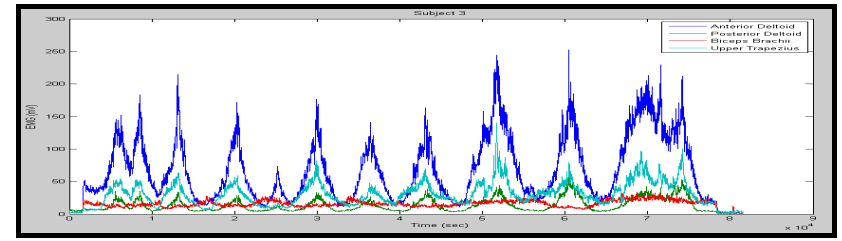

(c)

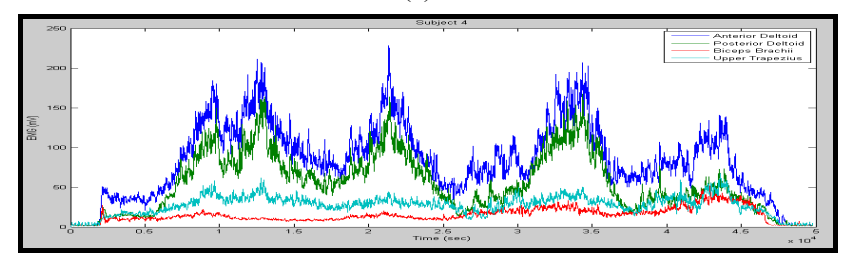

(d)

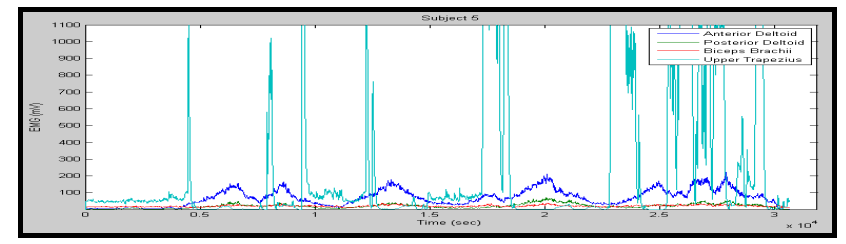

(e)

Figure 5. Muscle performance of (a) Subject 1 (b) Subject 2 (c) Subject 3 (d) Subject 4 and (e) Subject 5 during the usability test with ARIS

(Legend: Blue: Anterior Deltoid, Green: Posterior Deltoid, Red: Biceps Brachii, Cyan: Upper Trapezius)

contributed muscle to perform CME exercise followed by posterior deltoid and then upper trapeizus muscle. However the contribution of biceps brachii is very less in ARIS exercise. This information indicates that performing of CME exercise in ARIS strengthen the anterior deltoid, posterior deltoid and upper trapezius muscles. Therefore, it can be clearly seen that ARIS with biofeedback system achieves positive feedback and it has a potential to be used as a rehabilitation system although there are some rooms to improve according to the feedback from questionnaire.

\section{CONCLUSION AND FUTURE WORK}

In this paper, ARIS with biofeedback system which integrates with "Fool-the-Brain" concept, AR based rehabilitation exercise and biofeedback that aims for fast recovery of upper limb motor deficits with motivational approach had presented. The system has analyzed via
Heuristics evaluation and it showed encouraging results with positive feedback. This feedback indicates that the develop system is appropriate as a rehabilitation system for people with upper limb motor deficit. However, there are few improvements that require to be done according to the feedback from questionnaires and the work is underway to make these improvements. In addition, control method of VA model will be replaced with human intended movement by making use of recorded user's sEMG signals to become more effective rehabilitation system. After improvements have completed, clinical trial will be conducted in Port Kembla Rehabilitation Hospital in Australia and results will be reported in near future.

\section{REFERENCES}

[1] Physical Disability Australia. (2013, October). What is physical disability? Available: http://www.pda.org.au/what-is-physicaldisability

[2] H. Al-Issa, H. Regenbrecht, and L. Hale, "Augmented reality applications in rehabilitation to improve physical outcomes," Physical Therapy Reviews, vol. 17, pp. 16-28, 2012.

[3] Y. M. Aung and A. Al-Jumaily, "AR based upper limb rehabilitation system," in 4th IEEE RAS \& EMBS International Conference on Biomedical Robotics and Biomechatronics (BioRob), Rome, Italy, 2012, pp. 213-218.

[4] A. Dinevan, Y. M. Aung, and A. Al-Jumaily, "Human computer interactive system for fast recovery based stroke rehabilitation," in 11th International Conference on Hybrid Intelligent Systems (HIS), 2011, pp. 647-652.

[5] H. Huang, S. Wolf, and J. He, "Recent developments in biofeedback for neuromotor rehabilitation," Journal of NeuroEngineering and Rehabilitation, vol. 3, p. 11, 2006.

[6] Y. M. Aung and A. Al-Jumaily, "Shoulder rehabilitation with biofeedback simulation," in 2012 International Conference on Mechatronics and Automation (ICMA), Chengdu, China, 2012, pp. 974-979.

[7] Y. M. Aung and A. Al-Jumaily, "Neuromotor Rehabilitation System with Real-Time Biofeedback," International Journal of Computer Information Systems and Industrial Management Applications, vol. 5, pp. 550-556, 2013.

[8] K. C. Lin, Y. T. Chen, P. C. Huang, C. Y. Wu, W. L. Huang, H. W. Yang, H. T. Lai, and H. J. Lu, "Effect of mirror therapy combined with somatosensory stimulation on motor recovery and daily function in stroke patients: A pilot study," Journal of the Formosan Medical Association, 2012.

[9] H. Thieme, J. Mehrholz, M. Pohl, J. Behrens, and C. Dohle, "Mirror therapy for improving motor function after stroke," Stroke, vol. 44, pp. e1-e2, 2013.

[10] M. M. Lee, H.-y. Cho, and C. H. Song, "The Mirror Therapy Program Enhances Upper-Limb Motor Recovery and Motor Function in Acute Stroke Patients," American Journal of Physical Medicine \& Rehabilitation, vol. 91, pp. 689-700, 2012.

[11] H. Regenbrecht, S. Hoermann, G. McGregor, B. Dixon, E. Franz, C. Ott, L. Hale, T. Schubert, and J. Hoermann, "Visual manipulations for motor rehabilitation," Computers \& Graphics, vol. 36, pp. 819-834, 2012.

[12] P. Giraux and A. Sirigu, "Illusory movements of the paralyzed limb restore motor cortex activity," NeuroImage, vol. 20, Supplement 1, pp. S107-S111, 2003.

[13] Thought Techonology. (2013, October). FlexComp Infiniti. Available: http://www.thoughttechnology.com/

[14] Y. M. Aung and A. Al-Jumaily, "Illusion Approach for Upper Limb Motor Rehabilitation," in International Congress on Neurotechnology, Electronics and Informatics (NEUROTECHNIX 2013), Vilamoura, Algarve, Portugal, 2013, pp. 99-105. 
[15] H. Desurvire, M. Caplan, and J. A. Toth, "Using heuristics to evaluate the playability of games," in CHI '04 Extended Abstracts on Human Factors in Computing Systems, Vienna, Austria, 2004, pp. 15091512. 